\title{
A Simulation-based Performance COMPARISON OF THE MINIMUM NODE SIZE AND STABILITY-BASED CONNECTED DOMINATING SETS FOR MOBILE AD HOC NETWORKS
}

\author{
Natarajan Meghanathan \\ Jackson State University, Jackson, MS, USA \\ E-mail: natarajan.meghanathanejsums.edu
}

\begin{abstract}
The high-level contribution of this paper is a simulation-based comparison of two contrasting categories (minimum node size vs. stability) of connected dominating sets for mobile ad hoc networks. We pick the maximum density-based CDS (MaxD-CDS) and Node ID-based CDS (ID-CDS) to be representatives of the minimum node size-based CDS algorithms; the minimum velocity-based CDS (MinV-CDS) and the node stability index-based CDS (NSI-CDS) are chosen as representatives for stability-driven CDS. The MaxD-CDS, ID-CDS and MinV-CDS algorithms prefer to respectively include nodes with a larger number of uncovered neighbors, larger node ID and lower velocity into the CDS; the NSI-CDS algorithm prefers to include nodes with a larger value for the sum of the predicted expiration times of the links with the neighbor nodes. We simulate these algorithms under diverse conditions of node mobility and network density. We observe the MinV-CDS to be the most stable among all the CDSs as well as incur a lower hop count per path (through the CDS nodes) between any two nodes in the network. However, the MinV-CDS incurs a relatively larger CDS node size and edge size compared to the other three CDSs. Owing to a larger control overhead that could be incurred while broadcasting through a MinV-CDS (with larger number of CDS nodes and edges), the NSI-CDS can be considered as the best choice from the points of view of delay, energy, bandwidth and fairness of node usage.
\end{abstract}

\section{KEYWORDS}

Connected Dominating Sets, CDS Lifetime, CDS Node Size, Tradeoff, CDS Algorithms, Mobile Ad hoc Networks, Simulations

\section{INTRODUCTION}

A Mobile ad hoc network (MANET) is a category of wireless networks in which the topology changes dynamically with time due to arbitrary node movement. The MANET nodes have limited battery charge and hence operate with a restricted transmission range to conserve energy as well as avoid interference and collisions that may result from transmitting over longer distances. As a result, MANET routes are often multi-hop in nature and also temporally change depending on node mobility and availability. MANET routing protocols (for unicasting, multicasting and broadcasting) have been predominantly designed to be on-demand in nature rather than using a proactive strategy of determining the routes irrespective of the need [1][2].

The MANET on-demand routing protocols typically use a network-wide broadcast route-reply cycle (called flooding) to discover the appropriate communication topology (path, tree or mesh) [3]. A source node initiates the flooding of the Route-Request (RREQ) packets that propagate through several paths and reach the targeted destination (for unicasting) or receiver nodes (for multicasting). These nodes choose the path that best satisfies the principles of the routing protocol in use and respond with a Route-Reply (RREP) packet to the source on the selected 
route. With flooding, every node in the network is required to broadcast the RREQ packet exactly once in its neighborhood. Nevertheless, the redundancy of retransmissions and the resulting control overhead incurred with flooding is still too high, as every node spends energy and bandwidth to receive the RREQ packet from each of its neighbors.

A Connected Dominating Set (CDS) of a network graph comprises of a subset of the nodes such that every node in the network is either in the CDS or is a neighbor of a node in the CDS. Recent studies (e.g. [4][5][6][7][8]) have shown the CDS to be a viable backbone for networkwide broadcasting of a message (such as the RREQ message), initiated from one node. The message is broadcast only by the CDS nodes (nodes constituting the CDS) and the non-CDS nodes (who are neighbors of the CDS nodes) merely receive the message, once from each of their neighboring CDS nodes. The efficiency of broadcasting depends on the CDS Node Size that directly influences the number of redundant retransmissions. One category of CDS algorithms for MANETs aim to determine CDSs with reduced Node Size (i.e., constituent nodes) such that whole network could be covered as fewer nodes as possible. However, as observed in this paper and some of our previous work [9], such minimum node size-driven CDS have been observed to be unstable (i.e. have a limited lifetime). The other category of MANET CDS algorithms focus on maximizing the CDS Lifetime such that the control overhead incurred in frequently reconfiguring a CDS is reduced. Such stability-driven CDSs have been observed [9] to incur a larger Node Size and ratified through the simulation studies in this paper. We evaluate the tradeoff by calculating the ratio of the Node Size and Lifetime for the two categories of CDS algorithms.

We pick the maximum density-based CDS (MaxD-CDS) [10] and Node ID-based CDS (IDCDS) [11] to be representatives of the minimum node size-based CDS algorithms; the minimum velocity-based CDS (MinV-CDS) [12] and the node stability index-based CDS (NSI-CDS) [9] are chosen as representatives for stability-driven CDS. The NSI of a node is defined as the sum of the predicted expiration times of the links (LETs) with the neighbor nodes that are not yet covered by a CDS node; the NSI-CDS algorithm prefers to include nodes with larger NSI values into the CDS. The MaxD-CDS algorithm prefers to include nodes with the largest number of uncovered neighbors into the CDS; the ID-CDS algorithm prefers to include nodes with a larger node ID and having at least one uncovered neighbor into the CDS; and the MinV-CDS algorithm opts for slow moving nodes to be part of the CDS. We evaluate the four CDS algorithms under diverse conditions of network density and node mobility with respect to four metrics: CDS Lifetime, CDS Node Size, CDS Edge Size and the Hop Count per Path. To the best of our knowledge, we could not find such a comprehensive comparison study of the different CDS algorithms in the MANET literature.

We observe the MinV-CDS to incur a significantly larger lifetime compared to the other three CDSs as well as incur a lower hop count per path (through the CDS nodes) between any two nodes in the network. However, the MinV-CDS requires relatively more nodes to be part of the CDS to provide stability. Nevertheless, the Node Size-Lifetime tradeoff ratio is observed to be the lowest for the MinV-CDS for a majority of the scenarios. The rest of the paper is organized as follows: Section 2 presents a generic CDS algorithm that can be used to study each of the four CDS algorithms (MaxD-CDS, ID-CDS, MinV-CDS and NSI-CDS) that will be analyzed in the simulations. Section 3 presents the algorithm used to validate the existence of a currently known CDS at a particular time instant and decide on the need to determine a new CDS. Section 4 presents examples to illustrate the construction of the two minimum node size-based CDS (ID-CDS and MaxD-CDS). Section 5 presents examples to illustrate the construction of the two stability-based CDS (MinV-CDS and NSI-CDS). Section 6 presents the simulation results observed for these four CDSs and evaluates the Node Size - Lifetime tradeoff. Section 7 concludes the paper and describes future work. Throughout the paper, the terms 'vertex' and 'node', 'edge' and 'link', 'path' and 'route', 'packet' and 'message' are used interchangeably. They mean the same. 


\section{GENERIC CDS CONSTRUCTION ALGORITHM}

In this section, we present a generic algorithm that can be used to construct the four different CDSs, with appropriate changes in the data structure (Priority-Queue) used to store the list of covered nodes that could be the candidate CDS nodes and the use of the appropriate criteria in each of the iterations of the algorithm to evolve the particular CDS of interest. The advantage with proposing a generic algorithm is that all the four CDSs can be constructed with virtually the same time complexity.

\subsection{Network Model}

The network model used in this research is described as follows:

- We assume a homogeneous network of wireless nodes, each operating at a fixed transmission range, $R$.

- We use the unit-disk graph model [13] according to which there exists a link between any two nodes $i$ and $j$ at $\left(X_{i}, Y_{i}\right)$ and $\left(X_{j}, Y_{j}\right)$ in the network as long as the Euclidean distance $\sqrt{\left(X_{i}-X_{j}\right)^{2}+\left(Y_{i}-Y_{j}\right)^{2}}$ is less than or equal to the transmission range per node.

- The set of neighbors of a node $i$, Neighbors $(i)$, comprises of nodes that are connected to vertex $i$ in the unit-disk graph model.

- A node learns about its own location through location service schemes such as the Global Positioning System (GPS) [14] or any other scheme (e.g. [15]).

- A node learns the location and mobility parameters (velocity and direction of movement measured as the angle subscribed with respect to the positive $\mathrm{X}$-axis) of its neighbor nodes through the beacon messages periodically broadcast by their nodes in the neighborhood.

\subsection{Data Structures}

The data structures used by the CDS algorithm are as follows:

- CDS-Nodes-List: This list includes all the nodes that are part of the CDS

- Covered-Nodes-List: This list includes all the nodes that are either part of the CDS or is at least a neighbor node of a node in the CDS.

- Priority-Queue: This list includes all the nodes that are in the Covered-Nodes-List (but not in the CDS-Nodes-List) and are considered the candidate nodes for the next node to be selected for inclusion in the CDS-Nodes-List. The order in which the vertices are stored in the Priority-Queue varies with the CDS algorithms as described below. Any tie between the nodes is broken based on the node IDs (the contending node with a relatively larger ID is placed ahead of the other contending nodes).

- For the MaxD-CDS algorithm, the Priority-Queue stores the covered non-CDS nodes in the decreasing order of the node density (number of uncovered neighbors); the node with the largest number of uncovered neighbors is in the front of the queue.

- For the ID-CDS algorithm, the Priority-Queue stores the covered non-CDS nodes in the decreasing order of their node IDs and have at least one uncovered neighbor node; the node with the largest ID and has at least one uncovered neighbor node is in the front of the queue.

- For the MinV-CDS algorithm, the Priority-Queue stores the covered non-CDS nodes in the increasing order of the node velocities and have at least one uncovered neighbor node; the node with the lowest velocity and having at least one uncovered neighbor node is in the front of the queue.

- For the NSI-CDS algorithm, the Priority-Queue stores the covered non-CDS nodes in the decreasing order of the Node Stability Index (NSI, defined in Section 2.3) values; the node with the largest NSI value is in the front of the queue. 


\subsection{Auxiliary Variables}

- Node Stability Index: For the NSI-CDS algorithm, during a particular iteration, the Node Stability Index (NSI) value of a node is the sum of the predicted expiration times of the links (LETs) with the uncovered neighbor nodes. At any moment, every node maintains a LET-table comprising of the estimates of the LET values to each of its neighbor nodes based on the latest beacons received from the neighbor node. The predicted link expiration time (LET) of a link $i-j$ between two nodes $i$ and $j$, currently at $\left(X_{i}, Y_{i}\right)$ and $\left(X_{j}, Y_{j}\right)$, and moving with velocities $v_{i}$ and $v_{j}$ in directions $\theta_{i}$ and $\theta_{j}$ (with respect to the positive $\mathrm{X}$-axis) is computed using the formula proposed in [16]:

$$
\operatorname{LET}(i, j)=\frac{-(a b+c d)+\sqrt{\left(a^{2}+c^{2}\right) R^{2}-(a d-b c)^{2}}}{a^{2}+c^{2}} \ldots
$$

$$
\text { where } a=v_{i}^{*} \cos \theta_{i}-v_{j}^{*} \cos \theta_{j} ; b=X_{i}-X_{j} ; c=v_{i}^{*} \sin \theta_{i}-v_{j}^{*} \sin \theta_{j} ; d=Y_{i}-Y_{j}
$$

- CDS-Select-node: For every iteration, the candidate node (node $s$ in the pseudo code of Figure 1) selected for inclusion into the CDS-Node-List depends on the criteria used by each of the CDS algorithms.

- The CDS-Select-node for the MaxD-CDS algorithm is the covered non-CDS node with the largest node density (i.e., the largest number of uncovered neighbors).

- The CDS-Select-node for the ID-CDS algorithm is the covered non-CDS node with the largest ID and has at least one uncovered neighbor node.

- The CDS-Select-node for the MinV-CDS algorithm is the covered non-CDS node with the lowest velocity and has at least one uncovered neighbor node.

- The CDS-Select-node for the NSI-CDS algorithm is the node with the largest NSI value.

Note that the values for the density (the number of uncovered neighbors) and NSI (sum of the predicted LETs of the links with uncovered neighbors) for the nodes could change after the end of a particular iteration, depending on the updates to the CDS-Nodes-List and CoveredNodes-List; this would lead to rearrangement of the entire Priority-Queue at the end of each iteration of the MaxD-CDS and NSI-CDS algorithms. On the other hand, the values for the node velocity and node ID remain the same for each of the iterations of the MinV-CDS and ID-CDS construction algorithms on a particular instance of the network graph.

\subsection{Description of the Generic CDS Construction Algorithm}

The algorithm forms and outputs a CDS based on a given input graph representing a snapshot of the MANET at a particular time instant. Specifically, the algorithm outputs a list (CDS-NodeList) of all nodes that are part of the CDS formed based on the given MANET. The generic description and pseudo code given here (Figure 1) can be adapted for the particular CDS algorithm by appropriately incorporating the criteria for choosing the CDS-Select-node for each of the iterations as well as the criteria for maintaining the Priority-Queue during the different iterations of the CDS algorithm on a particular instance of the network graph.

The first node to be included in the CDS-Node-List is the CDS-Select-node ( $s$ ) chosen according to the criteria discussed in Section 2.3. A CDS node is considered to be "covered", so a CDS node is additionally added to the Covered-Nodes-List as it is added to the CDS-Node-List. All nodes that are adjacent to a CDS node are also said to be covered, so the uncovered neighbors of a CDS node are also added to the Covered-Nodes-List and to the Priority-Queue, as the node is added to the CDS-Node-List. The Priority-Queue for a particular CDS algorithm is maintained according to the criteria discussed in Section 2.2. The node at the front of the Priority-Queue is the next candidate node to become the CDS-Select-node, as long as the node satisfies the criteria mentioned in Section 2.3. 
If the Covered-Nodes-List does not contain all the vertices of the input graph and the PriorityQueue is not empty, we dequeue the Priority-Queue to extract a CDS-Select-node s that is not yet in the CDS-Node-List. All the uncovered neighbor nodes of node $s$ are added to the Covered-Nodes-List and to the Priority-Queue; node $s$ is also added to the CDS-Node-List. The above procedure is repeated until the Covered-Nodes-List contains all the vertices of the graph or the Priority-Queue becomes empty. If the Priority-Queue becomes empty and the CoveredNodes-List does not have at least one node that is present in the network graph, then the underlying network is considered to be disconnected.

The time complexity of the generic CDS algorithm can be given as: $\Theta((|E|+|V|) * \log |V|)$ on a network graph of $|V|$ nodes and $|E|$ edges - each of the $|V|$ nodes and their associated edges have to be explored for inclusion in the CDS. If the Priority-Queue is implemented as a binary heap, it takes $\Theta(\log |V|)$ time to reorganize the heap after each dequeue and enqueue operation. There could be a total of $|V|$ dequeue operations, one for each of the iterations and a total of $|E|$ enqueue operations, one for every edge that is traversed across all the iterations.

Input: Network Graph $G=(V, E)$, where $V$ is the set of vertices and $E$ is the set of edges $C D S$-Select-node $s$ - vertex chosen according to the selection criteria in Section 2.3

Auxiliary Variables and Functions: CDS-Node-List, Covered-Nodes-List, Priority-Queue, Neighbors(v) for every $v$ in $V$

Output: CDS-Node-List // contains the list of nodes part of the CDS.

Initialization:

Covered-Nodes-List $=\{s\} ;$ CDS-Node-List $=\Phi ;$ Priority-Queue $=\{s\}$

Begin Construction of CDS

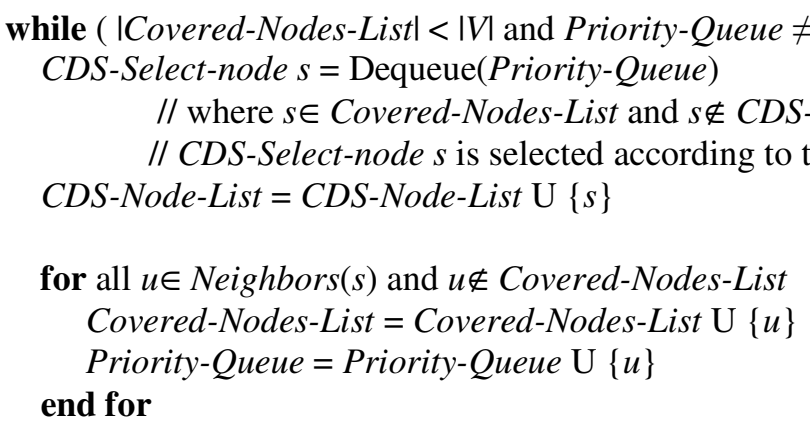

Rearrange the Priority-Queue according to the criteria for ordering the nodes

end while

if $(\mid$ Covered-Nodes-List $|<| V \mid$ and Priority-Queue $=\Phi)$ then

return NULL // the network is disconnected and there is no CDS covering all the nodes end if

return $C D S$-Node-List

End Construction of CDS

Figure 1: Generic Pseudo Code for CDS Construction 


\section{Algorithm to VAlidate THE Existence OF a CDS}

To reduce the control overhead, we intend to use a CDS as long as it exists and opt for a new CDS only if the currently known CDS ceases to exist. To determine whether a currently known CDS exists at a particular time instant, we construct a CDS-Edge-List comprising of edges that exist between any two nodes (according to the unit-disk graph model) in the CDS-Node-List known at that time instant. The two conditions that we test for are: (i) The CDS-induced graph comprising of the CDS-Node-List and the CDS-Edge-List should be connected and (ii) For every non-CDS node, there should be at least one CDS node as neighbor node. The pseudo code for the CDS validation algorithm is presented in Figure 2. Condition (i) is validated by running the classical Breadth First Search (BFS) algorithm [17] on the CDS-induced graph. Condition (ii) is validated only if condition (i) holds true. The algorithm returns true only if both the conditions hold true; otherwise, it returns false.

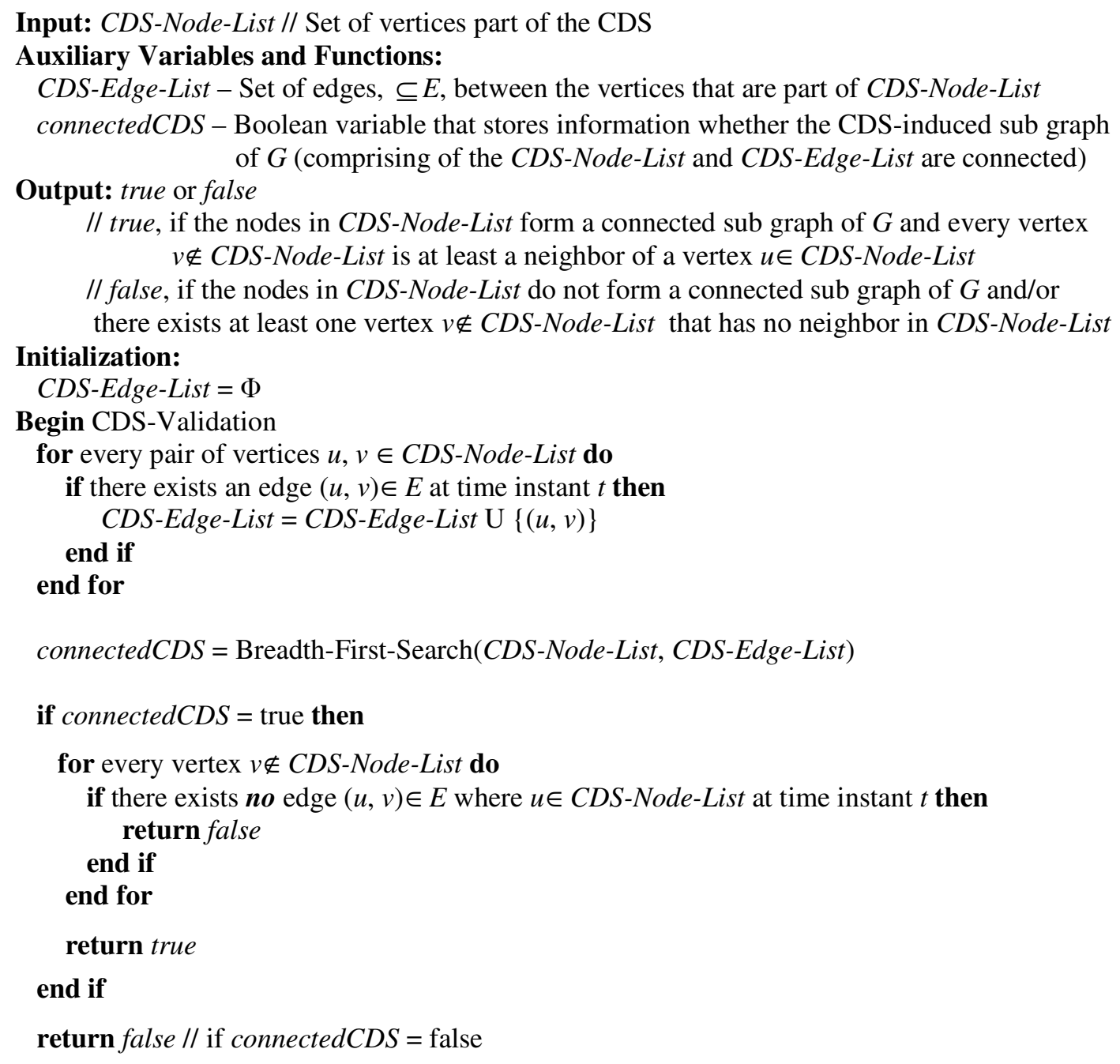

End CDS-Validation

Figure 2: Pseudo Code for the CDS Validation Algorithm 


\section{EXAMPLES FOR MAXD-CDS AND ID-CDS CONSTRUCTION}

We demonstrate the construction of the ID-CDS and MaxD-CDS through examples illustrated in Figures 4 and 5 respectively. As indicated in the legend (Figure 3) for these two figures wherein each circle indicates a node in the network graph, we represent: (i) a CDS node with a thick black-bordered circle that is gray-shaded inside; (ii) a covered node (a non-CDS node that is a neighbor of at least one CDS node) with a thick gray-bordered circle that has black-dots inside; (iii) a non-covered node (a node that is neither a CDS node nor a covered node) with a black thin circle with no shades or dots inside. In Figure 4 (illustrating the construction of the ID-CDS), the integer values inside each circle represents the node ID; in Figure 5 (illustrating the construction of the MaxD-CDS), the integer values outside of each circle represents the number of uncovered neighbors for the corresponding node. Both Figures 4 and 5 comprise of 16 nodes. The ID-CDS is constructed in 7 iterations and consists of 7 nodes and 8 edges; the MaxD-CDS is constructed in 5 iterations and also consists of 5 nodes and 5 edges.

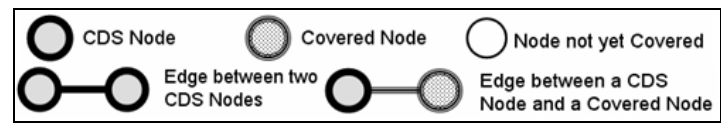

Figure 3: Legend for Figures 4 and 5

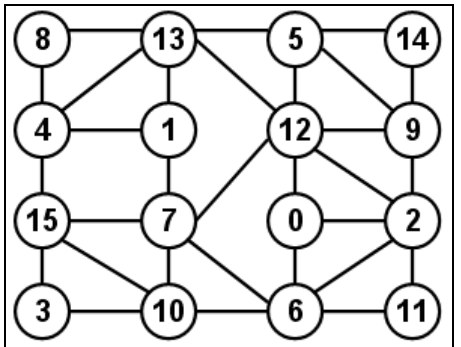

4.1: Initial Network Graph

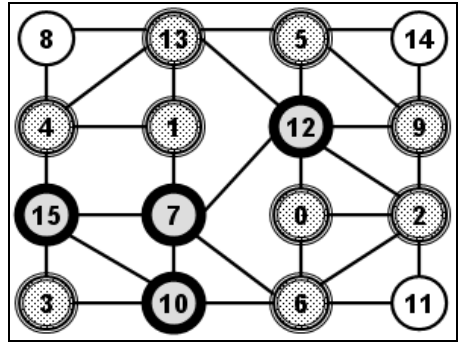

4.4: Iteration \# 6

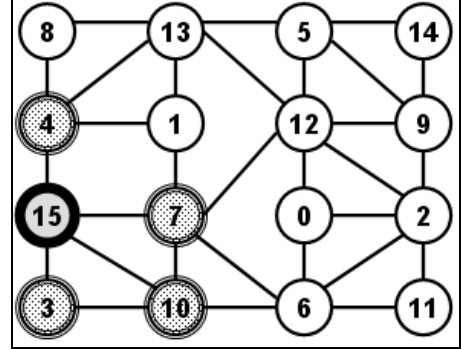

4.2: Iteration \# 1

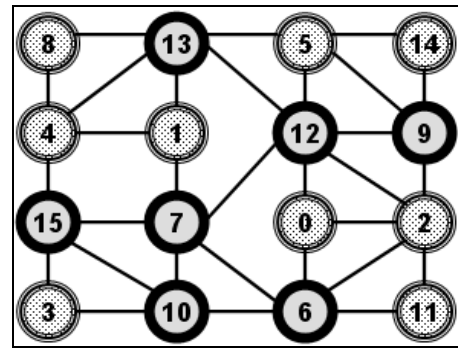

4.5: Iteration \# 7

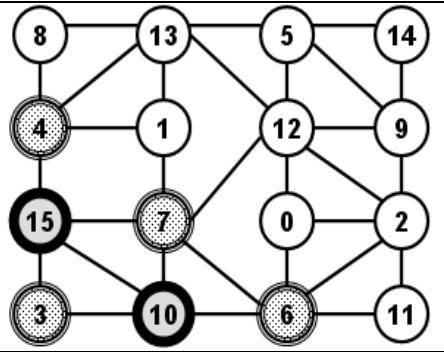

4.3: Iteration \# 2

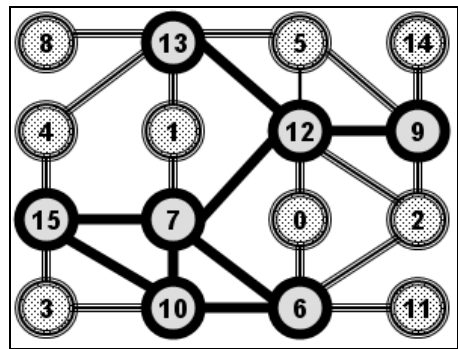

4.6: ID-CDS Sub Graph

Figure 4: Example to Illustrate the Construction of an ID-CDS

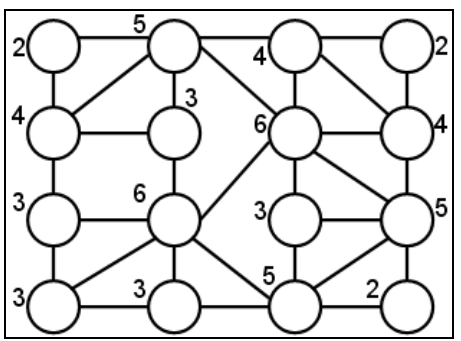

5.1: Initial Network Graph

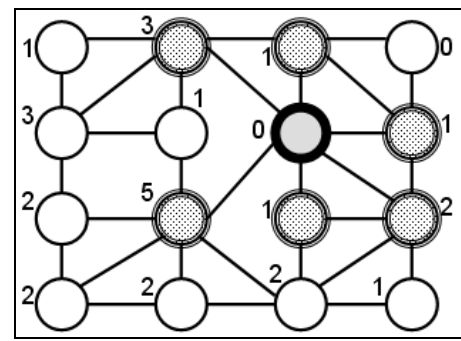

5.2: Iteration \# 1

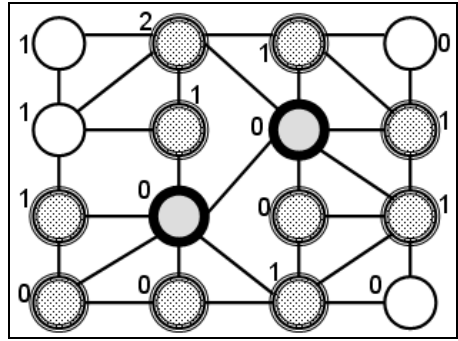

5.3: Iteration \# 2 


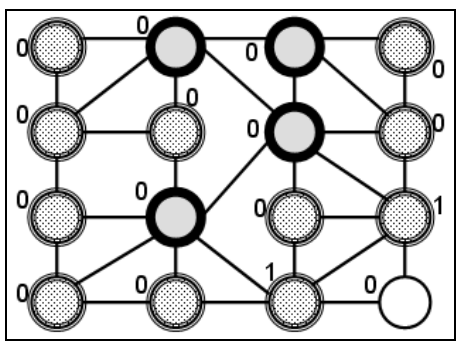

5.4: Iteration \# 4

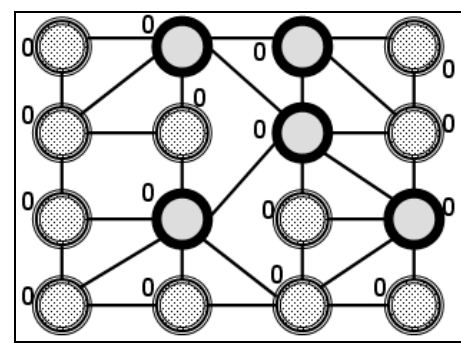

5.5: Iteration \# 5

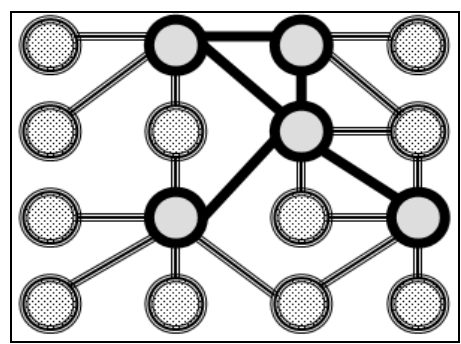

5.6: MaxD-CDS Sub Graph

Figure 5: Example to Illustrate the Construction of a Max. Density-based CDS (MaxD-CDS)

\section{EXAMPLES FOR MINV-CDS AND NSI-CDS CONSTRUCTION}

\subsection{Example to Construct the Minimum-Velocity-based CDS (MinV-CDS)}

We demonstrate the construction of the MinV-CDS through an example of a 24-node network illustrated in Figure 6 wherein each circle indicates a node. We represent: (i) a CDS node with a bold circle; (ii) a covered node (a non-CDS node that is a neighbor of at least one CDS node) with a shaded circle; (iii) a non-covered node (a node that is neither a CDS node nor a covered node) with a black thin circle that is neither shaded nor made bold. The integer values outside each circle represent the node ID; the integer values inside of each circle represent the number of uncovered neighbors for the corresponding node; the real-number inside the circle represents the node velocity (in $\mathrm{m} / \mathrm{s}$ ). As observed in Figure 6, the MinV-CDS is constructed in 13 iterations and consists of 14 nodes and 16 edges.

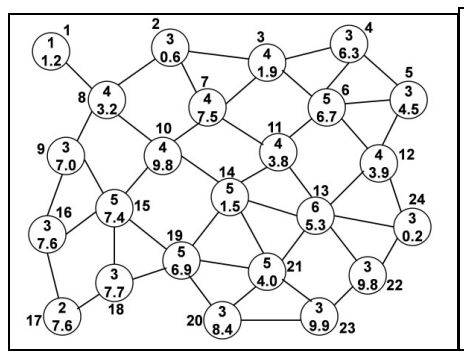

6.1: Initial Network

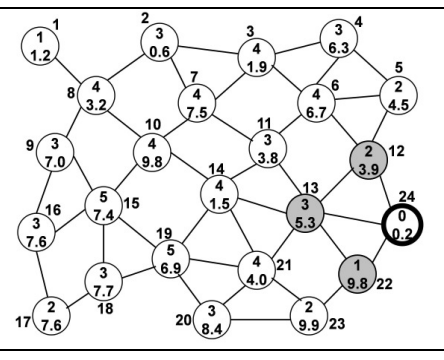

6.2: Iteration \# 1

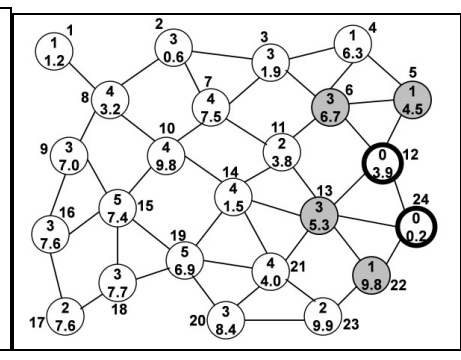

6.3: Iteration \# 2

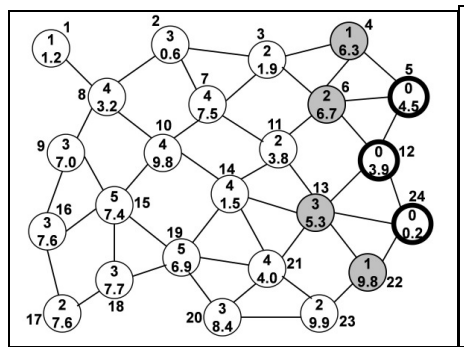

6.4: Iteration \# 3

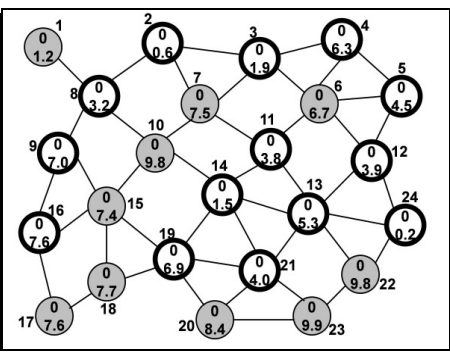

6.5: Final MinV-CDS

(at the end of Iteration \# 13)

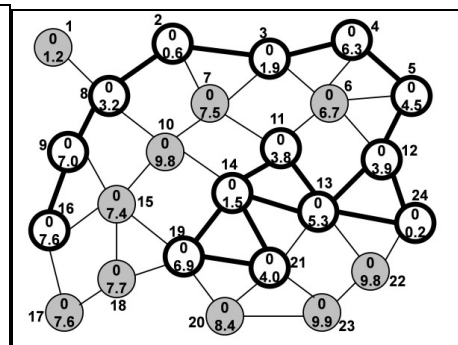

6.6: Final MinV-CDS Edges

Figure 6: Example to Illustrate the Construction of the Min. Velocity-based CDS (MinV-CDS) 


\subsection{Example to Construct the Node Stability Index-based CDS (NSI-CDS)}

We demonstrate the construction of the NSI-CDS through an example of a 24-node network illustrated in Figure 7 wherein each circle indicates a node. We represent: (i) a CDS node with a bold circle; (ii) a covered node (a non-CDS node that is a neighbor of at least one CDS node) with a shaded circle; (iii) a non-covered node (a node that is neither a CDS node nor a covered node) with a black thin circle that is neither shaded nor made bold. The integer values outside each circle represent the node ID; the real number on an edge represents the LET of the edge and the real number inside a circle represents the sum of the LETs of the edges incident on the node. As observed in Figure 7, the NSI-CDS is constructed in 10 iterations and consists of 10 nodes and 10 edges. On the NSI-CDS graph, the CDS edges (edges between two CDS nodes) are shown in bold thick lines; the edges between a CDS node and a non-CDS node are shown as regular lines.

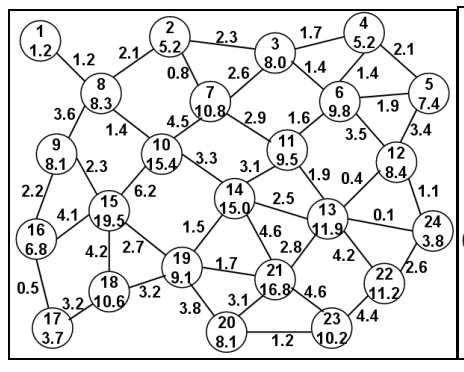

7.1: Initial Network

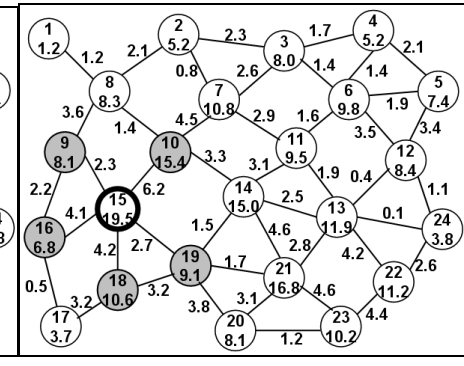

7.2: Iteration \# 1

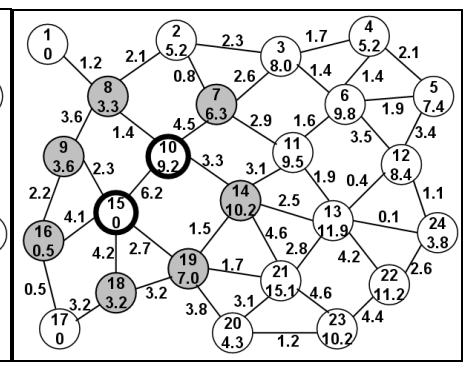

7.3: Iteration \# 2

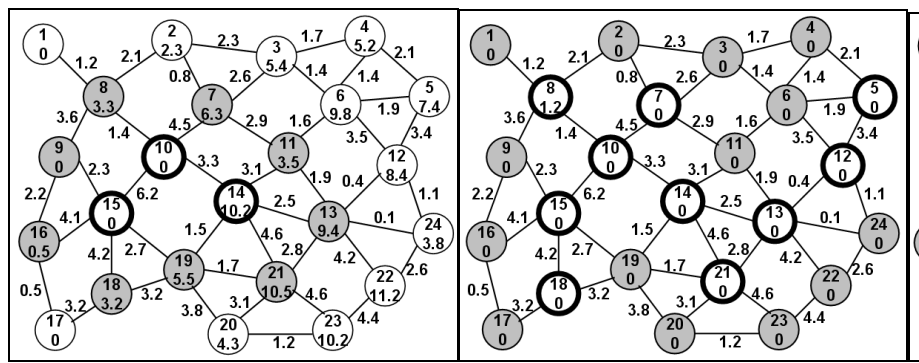

7.4: Iteration \# 3
7.5: Final NSI-CDS

(at the end of Iteration \# 10)

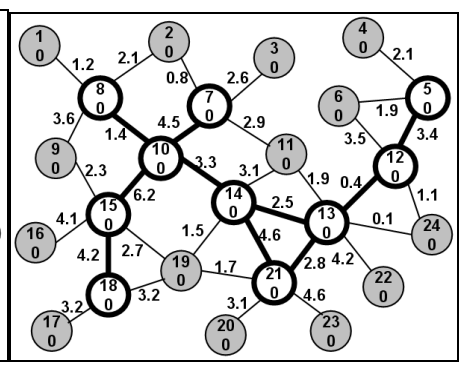

7.6: Final NSI-CDS Edges

Figure 7: Example to Construct the Node Stability Index-based CDS (NSI-CDS)

Compared to the MaxD-CDS (Figure 6) obtained on a 24-node network graph of the same topology, we observe the NSI-CDS to incur relatively fewer CDS nodes and CDS edges. This could be mainly attributed to the fact that the inclusion of a node into the NSI-CDS is decided by both the number of uncovered neighbors of the node as well as the LETs of the links to these uncovered neighbors. Nodes that have a larger number of uncovered neighbors are more likely to have a larger NSI value (the sum of the LETs of the links with these uncovered neighbors) and hence have greater chances for inclusion into the NSI-CDS. This characteristic of the NSICDS enables it to comprise of relatively fewer nodes as well as exist for a longer time.

Comparing Figures 4, 5, 6 and 7, the fraction of the total nodes in the network that become part of the CDS (in increasing order) is 5/16 (for MaxD-CDS), 10/24 (for NSI-CDS), 7/16 (for IDCDS) and 14/24 (for MinV-CDS). 


\section{SimULATIONS}

We used our own discrete-event simulator (developed in Java) that has also been successfully used in recent studies (e.g. [9][10][11][12]). We implemented all the four CDS algorithms (MaxD-CDS, ID-CDS, MinV-CDS and NSI-CDS) in the simulator.

\subsection{Network Density}

The dimensions of the network are $1000 \mathrm{~m} \times 1000 \mathrm{~m}$. Each node operates with a transmission range of $250 \mathrm{~m}$. The network density is measured as the ratio $N^{*} \pi R^{2} / A$, where $N$ is the number of nodes in the network, $R$ is the transmission range of a node and $A$ is the network area. We vary the network density by conducting simulations with 50 nodes (low density, corresponding to approximately 10 neighbors per node) and 100 nodes (high density, corresponding to approximately 20 neighbors per node).

\subsection{Node Mobility Model}

The well-known Random Waypoint model [18] is used as the mobility model for our simulations. With this model, each node moves independently, from one location to another randomly chosen location (within the network area) with a velocity uniform-randomly chosen from the range $\left[0, \ldots, v_{\max }\right]$. After reaching the targeted destination location, the node continues to move to another randomly chosen location with a different randomly chosen velocity from the same range. We simulated three levels of node mobility by varying the $v_{\max }$ value - low $\left(v_{\max }\right.$ $=5 \mathrm{~m} / \mathrm{s})$, moderate $\left(v_{\max }=25 \mathrm{~m} / \mathrm{s}\right)$ and high $\left(v_{\max }=50 \mathrm{~m} / \mathrm{s}\right)$.

\subsection{Simulation Strategy}

The following simulation strategy is adopted for each of the four CDS algorithms: For every combination of network density (50 and 100 nodes) and node mobility $\left(v_{\max }=5,25\right.$ and $50 \mathrm{~m} / \mathrm{s}$ ), we generate the mobility trace files for the nodes over a simulation time of 1000 seconds. We sample (take snapshots) the network topology for every 0.25 seconds. We construct the network graphs assuming a unit-disk graph model [13], according to which there exists a link between any two nodes in the network, if and only if the Euclidean distance between the two nodes is within the transmission range of $250 \mathrm{~m}$. If a CDS does not exist at a particular time instant, we run the appropriate CDS algorithm and tend to use the newly determined CDS as long as its exists (see Section 3 for the algorithm to check the existence of a CDS at any particular time instant). We repeat the above procedure for the entire simulation time of 1000 seconds.

To measure the hop count per path, we use a list 15 source-destination $(s-d)$ pairs and for each $s$ $d$ pair, we run the Breadth First Search (BFS) algorithm on a CDS-induced graph (i.e., the graph comprising of all the nodes in the network; there can be edges only between two CDS nodes and a CDS node to a non-CDS node) generated from the network snapshot for the particular time instant. The source/destination roles could be assigned to any node (CDS node or non-CDS node) in the network. A multi-hop $s-d$ path comprises of more than one hop wherein the intermediate nodes can be only CDS nodes. Paths between two non-CDS nodes are always multi-hop in nature, even if the two non-CDS nodes are neighbors of each other. However, if two CDS nodes or a CDS node and a non-CDS node are neighbors of each other, they can communicate directly.

\subsection{Performance Metrics}

The following performance metrics were measured in the simulations. Each metric value represented in Figures 8 through 12 represent average of values obtained for the CDS algorithms when simulated with 5 mobility trace files generated for every combination of network density and node mobility. 
- CDS Node Size: The time-averaged value for the number of constituent nodes of the connected dominating sets used across the entire simulation time period. For example, if we had used a sequence of three connected dominating sets of size 20, 18 and 25 nodes, existing for 10, 15 and 5 seconds respectively, then the time-averaged value for the CDS Node Size is $(20 * 10+18 * 15+25 * 5) /(10+15+5)=19.83$ and not 21 (the arithmetic average of 20,18 and 25).

- CDS Edge Size: The time-averaged value for the number of edges between the CDS nodes, considered for each of the connected dominating sets used across the entire simulation time.

- CDS Lifetime: The average of the duration of existence of a CDS across the entire simulation time period. The larger the CDS lifetime, the more stable it is.

- Hop Count per Path: The time-averaged value for the number of hops between sourcedestination $(s-d)$ pairs (15 $s-d$ pairs were considered) on paths determined on the CDSinduced network topologies determined for every time instant across the entire simulation time.

- CDS Node Size - Lifetime Tradeoff Ratio: A measure of the tradeoff between the two metrics - CDS Node Size and CDS Lifetime. We desire a smaller value for the CDS Node Size and a larger value for the CDS Lifetime. Hence, the smaller the ratio, we attribute the CDS to have effectively balanced both these metrics, without losing out significantly on either of them.

\subsection{CDS Node Size}

The MaxD-CDS and the MinV-CDS incur the lowest and largest values for the CDS Node Size. The only objective of the MaxD-CDS algorithm is to minimize the number of nodes constituting the CDS and it perfectly achieves this (the tradeoff being a significantly lower CDS lifetime, as discussed in Sections 6.7 and 6.8). The MinV-CDS and the ID-CDS algorithms have no way of controlling the number of nodes becoming part of the CDS. However, the ID-CDS does not incur a significantly larger CDS node size and achieves about the same CDS node size incurred by the NSI-CDS algorithm. The NSI-CDS is grouped under the category of stabilitybased algorithm because it is primarily designed to increase the lifetime of the CDS and it reasonably achieves that without substantially losing out on the CDS Node Size. On the other hand, the ID-CDS does not exist for a longer time and has to be frequently reconfigured. Since it does not incur significantly larger CDS node size and has a CDS Node Size - Tradeoff ratio (see Section 6.8) close to that of the MaxD-CDS, we group the ID-CDS under minimum node size-based CDS algorithms. While the ID-CDS and NSI-CDS require about $40-75 \%$ more CDS nodes (constituent nodes of the CDS) than the MaxD-CDS to cover the rest of the network nodes; the Node Size of MinV-CDS is 2.5 times or more than that of the MaxD-CDS. The MinV-CDS algorithm primarily prefers to include the slow moving nodes to be part of the CDS and its only secondary criteria is that the CDS-Select-node has to have at least one uncovered neighbor node. With such a design strategy, it is almost impossible to control the Node Size of a MinV-CDS. The NSI-CDS manages to have a controlled node size because the NSI of a node is defined as the sum of the predicted LETs of the links with the neighbor nodes. Nodes with more neighbors are more likely to have a larger NSI value and hence are likely to be included to the NSI-CDS; thus, in turn reducing the CDS Node Size.

For a given network density, the CDS Node Size incurred by the non-velocity-based algorithms (i.e., all the three algorithms other than the MinV-CDS) is not much sensitive to the node mobility. However, for MinV-CDS, we observe the Node Size for a MinV-CDS to increase with increase in node mobility, especially for networks of high density (100 nodes). For a given level of node mobility, the MaxD-CDS incurs a very minimal increase (at most 10\%) in the CDS Node Size as we double the number of nodes in the network. The ID-CDS and NSI-CDS incur a moderate increase $(15-25 \%)$ in the Node Size as we double the network density. The MinVCDS incurs the most significant increase (20-35\%) in the Node Size as we increase the number 
of nodes from 50 to 100: this could be attributed to the primary focus of the MinV-CDS algorithm being to include slow moving nodes into the CDS as long as they can cover at least one uncovered neighbor node.
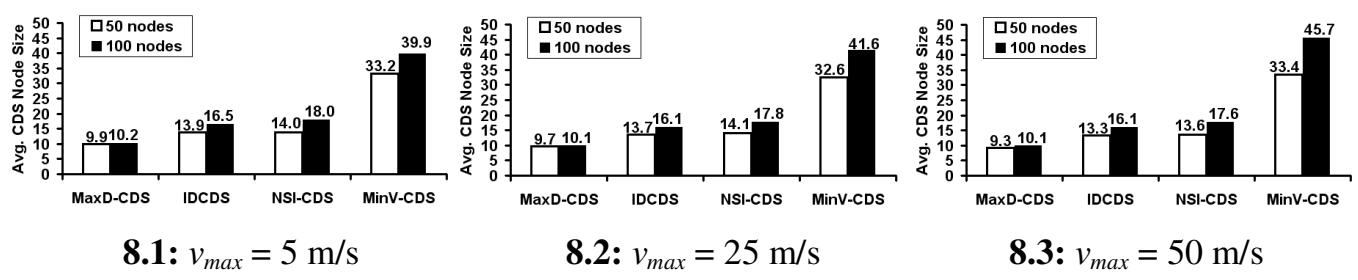

Figure 8: Average CDS Node Size

\subsection{CDS Edge Size}

By virtue of incurring a lower CDS Node Size, the MaxD-CDS also incurs a lower CDS Edge Size and the other three CDS algorithms are also ranked similar to their ranking observed for CDS Node Size. With the distance between the MaxD-CDS nodes being close to $80 \%$ or more of the transmission range at the time of CDS construction, the MaxD-CDS incurs relatively fewer edges and the number of MaxD-CDS edges and MaxD-CDS nodes are close enough to each other. In other words, the MaxD-CDS appears more like a tree; thus, the failure of even one or a couple of edges could easily disconnect the MaxD-CDS.
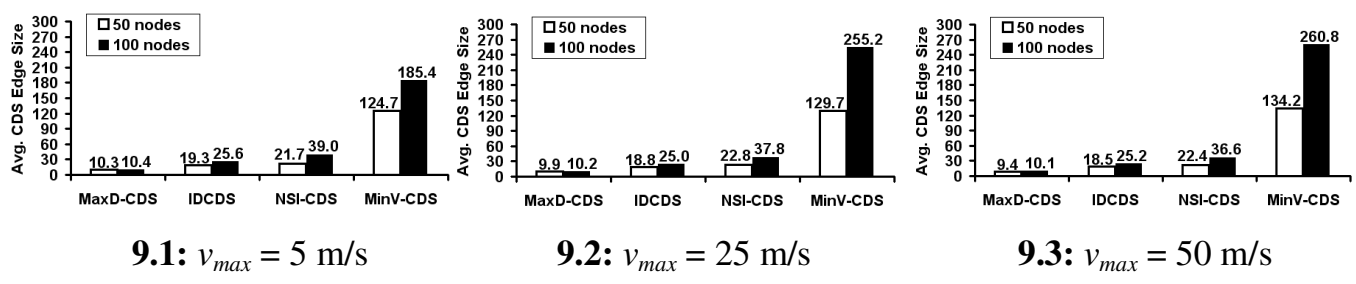

Figure 9: Average CDS Edge Size

As we include more nodes into the CDS, the number of edges between the constituent nodes of the CDS increases almost exponentially. While the ID-CDS and NSI-CDS incur an edge size that is within a factor of 2-4 to that of the MaxD-CDS, the MinV-CDS incurs an Edge Size that is 15-25 times more than that incurred with the MaxD-CDS. The percentage difference in the Edge Size for the NSI-CDS and ID-CDS for low-density networks is within $25 \%$. The presence of more edges between the CDS nodes has its own advantage and disadvantage: the advantage is that the CDS is more stable and does not get disconnected with a failure of one or more edges; the disadvantage is that broadcasting through the CDS nodes and edges introduces significant redundancy and control overhead. If one could incorporate a controlled broadcasting by selectively using only certain CDS edges; the rest of the CDS edges could be merely used as backup and be used for transmission and reception only when required to restore connectivity.

\subsection{CDS Lifetime}

The MaxD-CDS and MinV-CDS incur respectively the lowest and largest values for the CDS Lifetime. We observe the ID-CDS to yield 100-200\% longer lifetime than the MaxD-CDS and the NSI-CDS has in turn been observed to last 100-200\% longer than the ID-CDS; in other words, the lifetime of the NSI-CDS is about 200-400\% longer than that of the MaxD-CDS. We observe the lifetime of the MinV-CDS to be in turn 200-400\% longer than that of the NSI-CDS. Overall, with a mesh of edges, the ID-CDS and the stability-driven CDS (especially the MinVCDS) last significantly longer than that of the MaxD-CDS. The MaxD-CDS gets disconnected 
with any occasional failure of the edges. With the distance between the MaxD-CDS nodes being close to $80 \%$ or more of the transmission range at the time of CDS construction, the MaxD-CDS edges are highly susceptible to failure (i.e., the end nodes of the edge could move out of the transmission range of each other) at any time in the near future. The presence of multiple nodes and edges as part of the CDS contributes significantly to the lifetime of the MinV-CDS. Also, since the end nodes of the MinV-CDS edges are chosen to be moving with a lower velocity, these edges last for a relatively longer time compared to that of the fragile edges of the MaxDCDS.

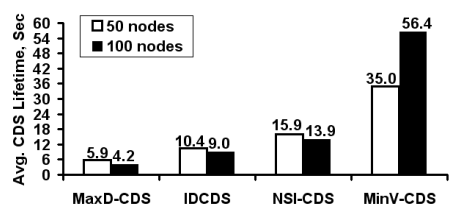

10.1: $v_{\max }=5 \mathrm{~m} / \mathrm{s}$

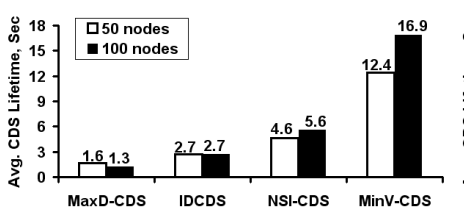

10.2: $v_{\max }=25 \mathrm{~m} / \mathrm{s}$

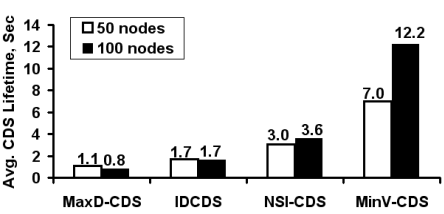

10.3: $v_{\max }=50 \mathrm{~m} / \mathrm{s}$

Figure 10: Average CDS Lifetime

For a fixed maximum node velocity, the lifetime of the MaxD-CDS decreases with increase in network density; whereas, the lifetime of the MinV-CDS increases with increase in network density. As the MaxD-CDS aims to cover all the nodes in the network (50 nodes or 100 nodes) with the fewest possible nodes as part of the CDS, the MaxD-CDS suffers relatively more disconnections in networks of higher density (wherein the MaxD-CDS Node Size increases by only at most $10 \%$ compared to low-density networks). On the other hand, the MinV-CDS accommodates more slow moving nodes that are available in high-density networks (i.e., incurs a $20-35 \%$ increase in the MinV-CDS Node Size) and is able to last relatively much longer compared to its duration of existence in low-density networks. The lifetime of the ID-CDS and NSI-CDS appears to remain the same or only marginally varies (i.e., a slight increase or decrease) with changes in the network density.

\subsection{Hop Count per Path}

We observe the hop count of the paths on the CDS-induced graphs of 50 and 100 nodes to be close to 3.0 or slightly above. The ID-CDS and the MinV-CDS respectively incur the largest and lowest values for the hop count per path. The relatively lower hop count per path when using the MinV-CDS can be attributed to the presence of a larger number of constituent nodes and edges as part of the CDS. As a result, it is possible to determine the $s$ - $d$ path with a hop count closer to that of the minimum hop count for the source-destination pairs. On the other hand, with a limited number of edges on the CDS-induced graphs, the ID-CDS and also the MaxD-CDS sustain a relatively larger hop count per path. However, the difference in the magnitude of the hop counts per path is within $15 \%$. Nevertheless, paths with lower hop count are usually preferred for delay-sensitive applications as well as when operating the network with energy-constrained nodes. The more the intermediate nodes on a path, the more the energy consumed across the network as well as the delay suffered by the packets sent on the multi-hop paths. In this context, the MinV-CDS paths (that also exist longer due to the longer lifetime of the MinV-CDS) are more preferable over the other connected dominating sets.

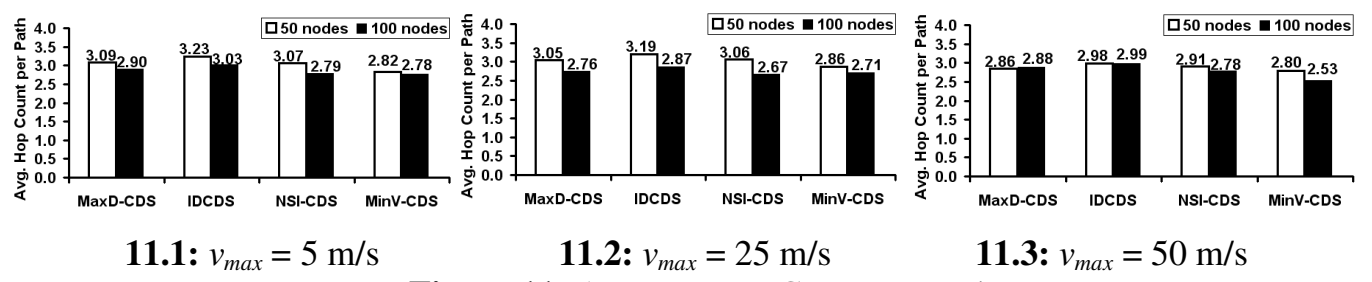

Figure 11: Average Hop Count per Path 
In addition to the drawbacks of longer delay and larger network-wide energy consumption, multi-hop paths with a larger hop count on CDS-induced graphs involving a fewer candidate intermediate nodes can be unfair to these CDS nodes. Nodes that are part of these connected dominating sets could be relatively heavily used compared to the non-CDS nodes. This could lead to premature failure of critical nodes, especially those located in the center of the network, resulting in loss of network connectivity, especially in low-density networks. On the other hand, when several CDS nodes are available to serve as the intermediate nodes on multi-hop paths, we could fairly distribute the routing load across several nodes; this could in turn enhance the fairness of node usage as well as help to incur a relatively lower end-to-end delay per data packet due to the reduced forwarding load at these nodes.

\subsection{CDS Node Size - Lifetime Tradeoff Ratio}

As we observe the stability-driven connected dominating sets (MinV-CDS and NSI-CDS) to sustain a significantly longer lifetime (compared to the MaxD-CDS and ID-CDS) at the expense of a larger CDS Node Size, we evaluate the tradeoff between the CDS Node Size and CDS Lifetime by calculating the ratio of these two metrics (Node Size/Lifetime) for the four connected dominating sets. Since we prefer to have a lower CDS Node Size and a larger CDS Lifetime, connected dominating sets that incur a lower value for the Node Size/Lifetime ratio can be attributed to effectively balance the two metrics.

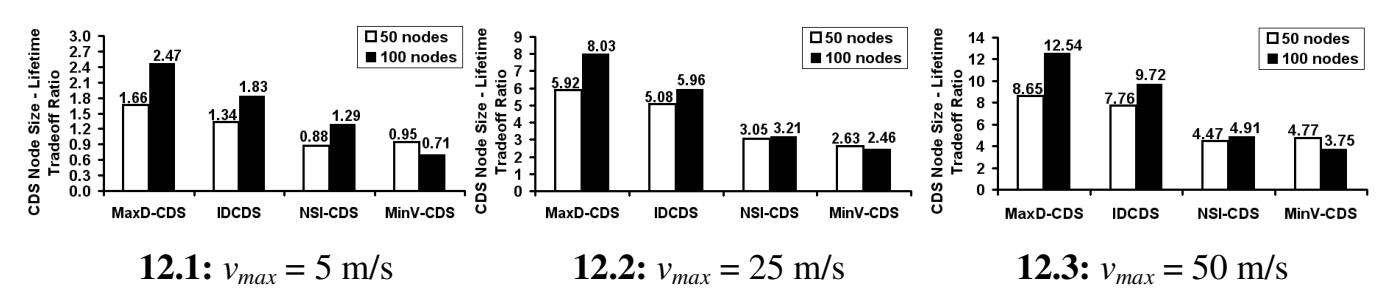

Figure 12: CDS Node Size - Lifetime Tradeoff Ratio

Incidentally, as observed in Figure 12, the MinV-CDS is observed to have the lowest value for this tradeoff ratio. The MinV-CDS sustains a lifetime that is 7-15 times more than the lifetime of the MaxD-CDS, especially in high-density networks and the above said gain in the lifetime is incurred with a Node Size that is at most 2.5 times more than that of the MaxD-CDS. However, such a large number of nodes and the resulting edges may not be preferable from the point of view of the control overhead incurred for any broadcast communication on the CDS-induced graph. In this context, the NSI-CDS can be preferred for stable communication with only a very moderately large control overhead. The NSI-CDS has a tradeoff ratio that is very much comparable to that of the MinV-CDS. The lifetime of NSI-CDS is 2-4 times more than the lifetime of the MaxD-CDS and the Node Size is at most 40-75\% more than that of the MaxDCDS. The hop counts per path determined for the NSI-CDS (at most 5\% larger than that of the hop counts per path using MinV-CDS) are also typically lower than that of the hop counts per path determined using the MaxD-CDS and ID-CDS.

\section{CONCLUSIONS AND FUTURE WORK}

We evaluated the performance of two representatives from each of the two categories of minimum-node size driven and stability-driven connected dominating sets (CDS) for MANETs. The minimum velocity-based MinV-CDS has been observed to yield the largest lifetime, the lowest hop count per path as well as the lowest CDS Node Size/Lifetime tradeoff ratio. The minimum-node size based MaxD-CDS and ID-CDS are quite unstable (especially the MaxDCDS) as well as incur a larger hop count per path. However, if the control overhead incurred in broadcasting through a CDS with a relatively larger number of nodes and edges is a concern, the 
Node Stability Index based CDS (NSI-CDS) can be considered the best choice: the NSI-CDS sustains a significantly lower tradeoff ratio and lower hop count per path (comparable to that of the MinV-CDS) and at the same time does not incur a significantly larger Node Size. This way, the NSI-CDS is also the most preferred candidate from the points of view of optimal energy consumption, delay per path, bandwidth and fairness of node usage.

In addition to the detailed simulation-based evaluation of the four CDS algorithms (Maximum density-based MaxD-CDS, Node ID-based ID-CDS, Minimum velocity-based MinV-CDS and the Node Stability Index-based NSI-CDS), we present a generic algorithm (in the first half of the paper) that can be used to effectively implement each of these four CDS algorithms by appropriately managing the Priority-Queue (according to the criteria used by the individual algorithms) to store the candidate covered nodes that can be chosen for inclusion to the CDS. This way, the above four connected dominating sets can be determined with virtually the same time complexity.

As part of future work, we will attempt to study the four CDS algorithms with respect to mobility models other than the Random Waypoint model as well as study the applicability of the connected dominating sets for multicast communication. We will also study the energy consumption and fairness of node usage (measured through the node lifetime) with respect to routing (unicast and multicast) using the CDS-induced graphs vis-à-vis the unicast and multicast routing protocols proposed for MANETs.

\section{REFERENCES}

[1] J. Broch, D. A. Maltz, D. B. Johnson, Y. C. Hu and J. Jetcheva, "A Performance Comparison of Multi-hop Wireless Ad hoc Network Routing Protocols," Proceedings of the $4^{\text {th }}$ Annual International Conference on Mobile Computing and Networking, pp. 85-97, October 1998.

[2] P. Johansson. T. Larson, N. Hedman, B. Mielczarek and M. DegerMark, "Scenario-based Performance Analysis of Routing Protocols for Mobile Ad hoc Networks," Proceedings of the $5^{\text {th }}$ Annual ACM/IEEE International Conference on Mobile Computing and Networking, pp. 195-206, August 1999.

[3] C. Siva Ram Murthy and B. S. Manoj, Ad Hoc Wireless Networks: Architectures and Protocols, Prentice Hall, $1^{\text {st }}$ Edition, June 2004.

[4] P. Sinha, R. Sivakumar and V. Bhargavan, "Enhancing Ad hoc Routing with Dynamic Virtual Infrastructures," Proceedings of the $20^{\text {th }}$ Annual Joint Conference of the IEEE Computer and Communications Societies (INFOCOM), vol. 3, pp. 1763 -1772, 2001.

[5] F. Wang, M. Min, Y. Li and D. Du, "On the Construction of Stable Virtual Backbones in Mobile Ad hoc Networks," Proceedings of the IEEE International Performance Computing and Communciations Conference (IPCCC), 2005.

[6] K. Sakai, M.-T. Sun and W.-S. Ku, "Maintaining CDS in Mobile Ad hoc Networks," Wireless Algorithms Systems and Applications, Lecture Notes in Computer Science, vol. 5258, pp. 141-153, October 2008.

[7] P.-R. Sheu, H.-Y. Tsai, Y.-P. Lee and J. Y. Cheng, "On Calculating Stable Connected Dominating Sets Based on Link Stability for Mobile Ad hoc Networks," Tamkang Journal of Science and Engineering, vol. 12, no. 4, pp. 417-428, 2009.

[8] L. Bao and J. J. Garcia-Luna-Aceves, "Stable Energy-aware Topology Management in Ad hoc Networks," Ad hoc Networks, vol. 8, no. 3, pp. 313 - 327, May 2010.

[9] N. Meghanathan, "A Node Stability Index-based Connected Dominating Set Algorithm for Mobile Ad hoc Networks," The $3^{\text {rd }}$ International Conference on Wireless \& Mobile Networks, LNICST 84, pp. 253-262, January 2012. 
International Journal of Computer Networks \& Communications (IJCNC) Vol.4, No.2, March 2012

[10] N. Meghanathan, "An Algorithm to Determine the Sequence of Stable Connected Dominating Sets in Mobile Ad hoc Networks," Proceedings of the $2^{\text {nd }}$ Advanced International Conference on Telecommunications, Guadeloupe, French Caribbean, February 2006.

[11] N. Velummylum and N. Meghanathan, "On the Utilization of ID-based Connected Dominating Sets for Mobile Ad hoc Networks," International Journal of Advanced Research in Computer Science , vol. 1, no. 3, pp. 36-43, September-October 2010.

[12] N. Meghanathan, "Use of Minimum Node Velocity Based Stable Connected Dominating Sets for Mobile Ad hoc Networks," International Journal of Computer Applications: Special Issue on Recent Advancements in Mobile Ad hoc Networks, vol. 2, pp. 89-96, September 2010.

[13] F. Kuhn, T. Moscibroda and R. Wattenhofer, "Unit Disk Graph Approximation," Proceedings of the Workshop on the Foundations of Mobile Computing (DIALM-POMC), pp. 17-23, October 2004.

[14] B. Hofmann-Wellenhof, H. Lichtenegger and J. Collins, Global Positioning System: Theory and Practice, $5^{\text {th }}$ ed., Springer, September 2004.

[15] W. Keiss, H. Fuessler and J. Widmer, "Hierarchical Location Service for Mobile Ad hoc Networks," ACM SIGMOBILE Mobile Computing and Communications Review, vol. 8, no. 4, pp. 47-58, 2004.

[16] W. Su, S-J. Lee and M. Gerla, "Mobility Prediction and Routing in Ad hoc Wireless Networks," International Journal of Network Management, vol. 11, no. 1, pp. 3-30, 2001.

[17] T. H. Cormen, C. E. Leiserson, R. L. Rivest and C. Stein, Introduction to Algorithms, $3^{\text {rd }}$ Edition, MIT Press, September 2009.

[18] C. Bettstetter, H. Hartenstein and X. Perez-Costa, "Stochastic Properties of the Random-Way Point Mobility Model,” Wireless Networks, pp. 555-567, vol. 10, no. 5, September 2004. 